\title{
Modelling and Testing of the Application Designed for Ballistic Calculations of Detected Objects
}

\author{
Artur Karapetyan 1, *, Aram Ter-Martirosyan ${ }^{2}$, Beniamin Badalyan ${ }^{1}$, Armand Karapetyan ${ }^{3}$ \\ ${ }^{1}$ Radio Devices, University of NPUA (National Polytechnic University of Armenia), Yerevan, Armenia \\ ${ }^{2}$ AESA (Armenian Engineers and Scientists of America), Glendale, USA \\ ${ }^{3}$ Information Security and Software, University of NPUA (National Polytechnic University of Armenia), Yerevan, Armenia
}

\section{Email address:}

artur.karapetyan@rocketmail.com (A. Karapetyan), aram@connectto.com (A. Ter-Martirosyan), agentben@mail.ru (B. Badalyan), karapetyan.armand@mail.ru (A. Karapetyan)

${ }^{*}$ Corresponding author

\section{To cite this article:}

Artur Karapetyan, Aram Ter-Martirosyan, Beniamin Badalyan, Armand Karapetyan. Modelling and Testing of the Application Designed for Ballistic Calculations of Detected Objects. Journal of Electrical and Electronic Engineering. Vol. 8, No. 1, 2020, pp. 16-20.

doi: $10.11648 /$ j.jeee.20200801.13

Received: February 24, 2019; Accepted: April 25, 2019; Published: January 6, 2020

\begin{abstract}
Detection of objects is a widespread problem; the proposed solutions differ from one another depending on the sphere of application. However, the discovery of objects is only a part of the ultimate goal; the main aim can be the pursuit of the discovered object, the determination of the law of motion and striking of it. The research comprehensively discusses "AYA Sport" application, developed by us, designed for determination of the position of the object and its further striking. It also reviews the most widely used systems for the solution of the above-mentioned problem presenting both their positive and negative aspects.
\end{abstract}

Keywords: Object Detection Systems, Object Striking Systems, Ballistic Calculator, Strelok, AYA Sport

\section{Introduction}

Today, along with technological growth, remote and selfpropelled mobile objects are developing. The sharp increase of the latter and often their uncontrolled distribution cause violation of the territorial integrity of a person or state, illegal tracking of people and various other violations of the law. To avoid such problems, it is necessary to reconsider the approach of detecting the violation causing object and, where appropriate, to neutralize it. At this moment, RADAR and LiDAR systems are already being widely used. The aforementioned equipment enables to detect objects in a space, as well as determine their direction of movement and calculate the law of movement. However, experience shows that using RARAR or LiDAR systems, which have a fairly high cost, is not suitable for all cases. At this moment, the socalled "Camera Vision" system is often being used, which, having extremely low cost, shows a fairly high degree of accuracy, depending on the artificial intelligence used in the system and the technical parameters of the camera.
However, the detection of objects is only a part of the problem, as it may be necessary to strike them after detection. To solve this problem, ballistic calculators are frequently used, which take on that time-consuming and sometimes even complicated task of calculating. In case of using such calculators, it is possible to perform ballistic calculations in just a few seconds. However, at present moment, there are quite a lot and significant omissions in similar calculators, the first of which is that they are not automated. In other words, it is necessary for a person to interact, which, in fact, leads to a slowing down of the process and a possible miscalculation.

The purpose of the paper is to present the most common solution offering programs for ballistic calculations, including fully automated "AYA Sport", designed by us, introducing their main characteristics. Also, with the use of the designed application, the projecting of the conceptual model of the fully automated system, designed for the complete solution of the main problem, namely the detection, separation and, if necessary, striking of the objects, is being 
done in the work.

\section{Restrictions of Existing Applications}

At present, the Russian Strelok software application is widely distributed with its "Strelok +" and "Strelok Pro" versions [1]. It allows shooting and striking the required object precisely by performing ballistic calculations. Both versions allow the user to get the necessary corrections of the target for striking it by entering the wind direction and speed, the type of weapon, the air humidity, the pressure, the temperature, and the fire conditions.

These applications, as opposed to their competitors available on the market, give the opportunity to automatically get the wind direction and speed from external devices if BLUETOOTH system is available.

In addition, it should be noted that the "Strelok + " and "Strelok Pro" software contain quite a large number of bullets and signs.

The benefits of Strelok + and Strelok Pro are:

1. High accuracy of calculations;

2. Opportunity to get wind direction and speed from external devices.

Deficiencies of the applications are:

1. Uncomfortable and complicated interface,

2. Necessity to enter input parameters manually,

3. It takes a lot of time to get the calculation results.

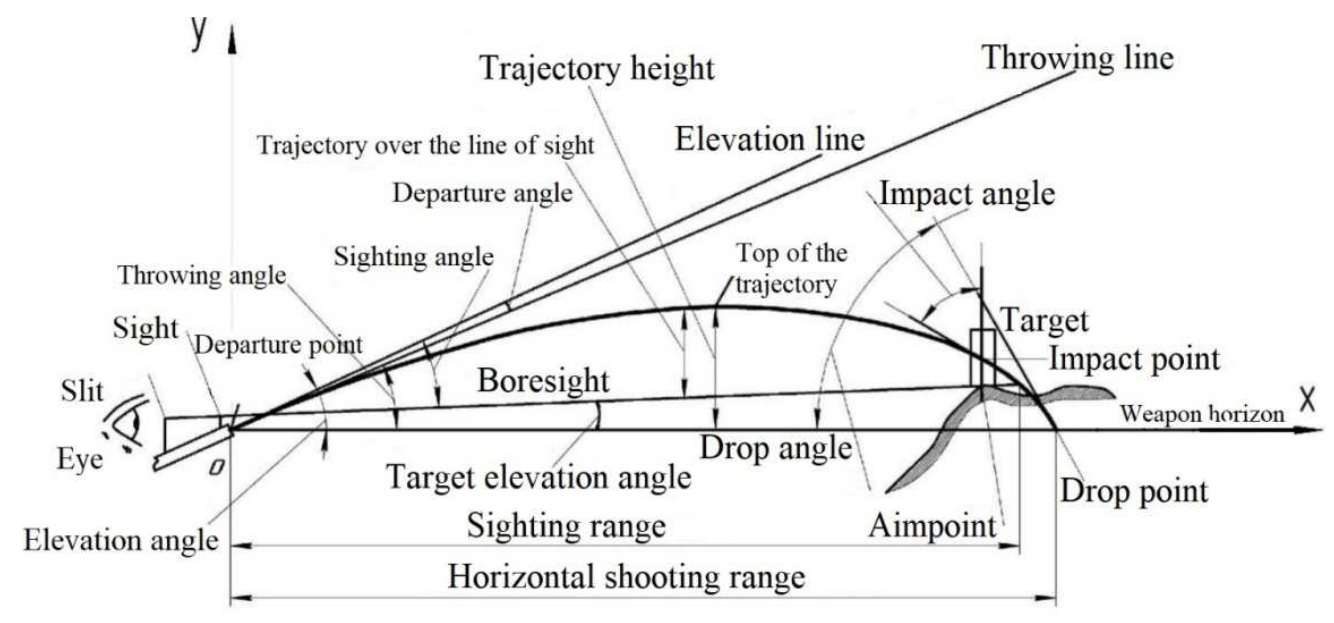

Figure 1. Bullet flight trajectory.

\section{The Developed App's Description}

"AYA Sport" is based on the schematic image of figure 1 [2-6]:

To perform ballistic calculations, the following parameters are needed:

$\mathrm{V}_{0}$ - the initial bullet velocity,

$\mathrm{d}-$ the bullet diameter,

$\mathrm{q}$ - the bullet mass,

$\mathrm{i}$ - the drag coefficient of the bullet, depending on its shape,

$\mathrm{x}$ - the distance to the destination point.

The following formulas system is used when making calculations: (1-6)

$$
\begin{aligned}
\frac{d v}{d x} & =\frac{1}{V \cos \theta}\left(-\frac{F}{q}-g \sin \theta\right), \\
\frac{d y}{d x} & =\tan \theta, \\
\frac{d \theta}{d x} & =-\frac{g}{V^{2}} \\
\frac{d t}{d x} & =-\frac{1}{V \cos \theta} \\
F=\frac{\rho V^{2}}{2} & * \frac{\pi d^{2}}{4} * H(y) * C_{X}(M),
\end{aligned}
$$

$$
M=\frac{V}{a}
$$

where $\mathrm{F}$ - is the force of aerodynamic resistance, $\mathrm{Cx}(\mathrm{M})$ - the front resistance coefficient, $\mathrm{M}$ - the mach number, $\mathrm{a}-$ the speed of sound, $\mathrm{H}(\mathrm{y})$ - the function of atmospheric density, $\rho$ - air density.

As a result of calculations the following data are obtained:

$\mathrm{V}(\mathrm{x})$ - muzzle velocity(the speed of the bullet at the moment),

$y(x)$ - the height of the trajectory of the weapon towards horizon,

$\mathrm{t}(\mathrm{x})$ - flight time,

$\theta(\mathrm{x})$ - the angle formed between the horizon and the trajectory.

When designing this application, the emphasis has been put on getting the most convenient and simple interface, fast and automatic data acquisition. As a result, with only one touch you can get an accurate ballistic calculation. The main window of "AYA Sport" has the look of Figure 1. [7]: 


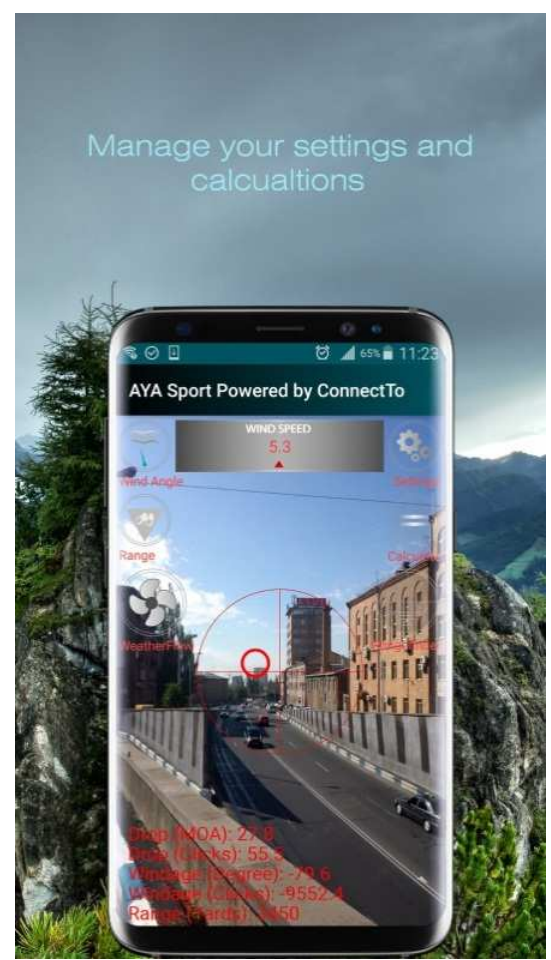

Figure 2. The main window of "AYA Sport."

As can be seen from the Figure 2, the main window has an extremely intuitive interface.

Unlike all the existing competing programs, "AYA Sport" enables users to activate the automatic data transmission from the wind direction, speed and distance measurement devices from its main window.

In addition to receiving wind parameters from external devices, it can be done manually, as shown in Figure 3.

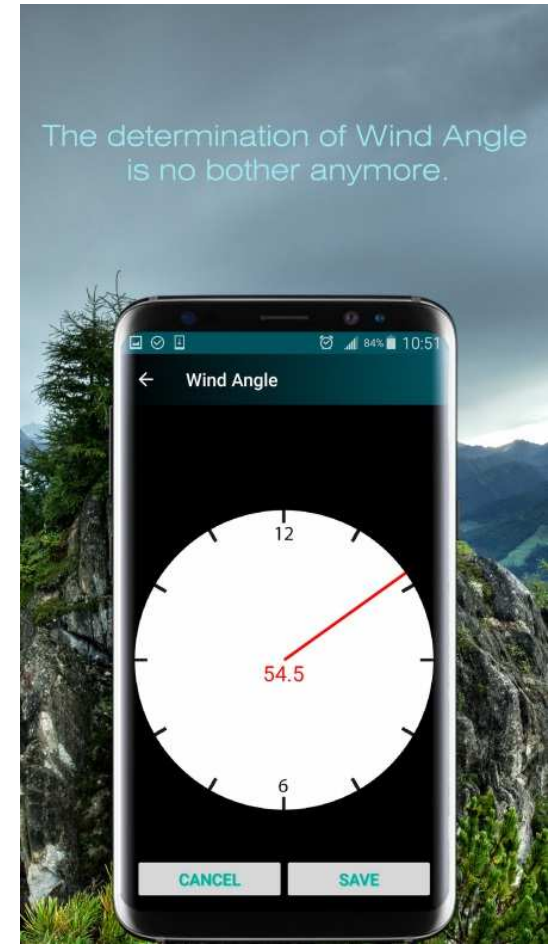

Figure 3. Manual input window of wind direction.
In the event of distance, the user has more options, in addition to receiving automatic information from the remote, it is possible to get it by using GoogleMaps, an integrated distance measuring application (Figure 4), or type it manually.

GoogleMaps is a well-known and widely-used software, and the integrated distance measuring application used in "AYA Sport" presents a mathematical solution to the problem.

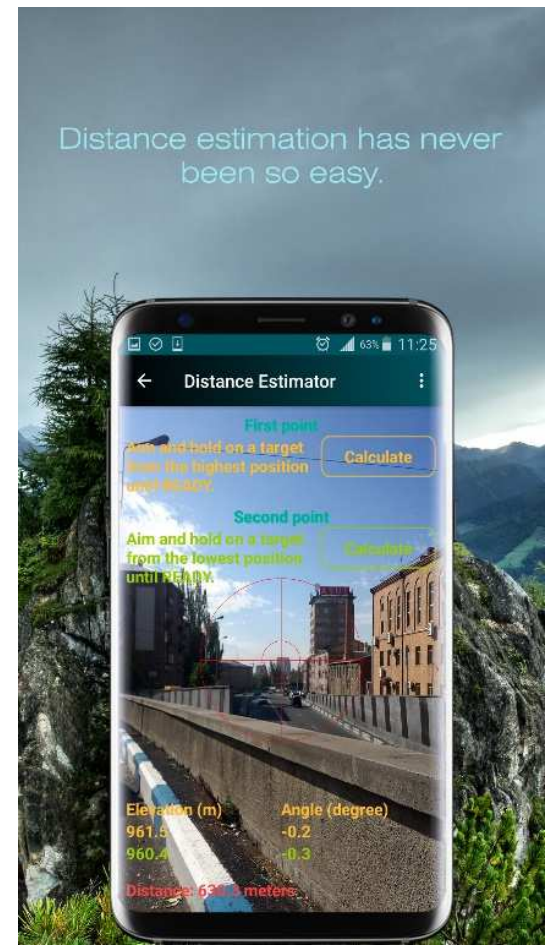

Figure 4. Integrated distance measuring application.

Calculations are based on the following logic: the distance (h) between the first (A) and the second (B) observation points is determined by using the sensors integrated in the smartphone.

Then by dividing $\mathrm{h}$ into the difference between the tangents of the angles formed between the horizon and each observation point and the horizon and the target $(\mathrm{T})$, the projection $(p)$ of the connecting segment of the observation point and the target is obtained (7).

$$
p=\frac{h}{\tan \alpha-\tan \beta} \text {. }
$$

The diagram is shown in Figure 5

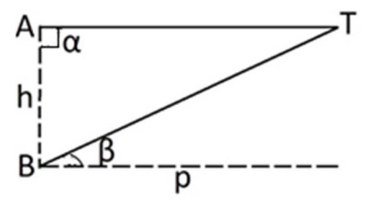

Figure 5. The diagram of distance detection

It should be noted that "AYA Sport" software contains a substantial database of weapon bullets and sign, which, based 
on the user's preferences, can also be replenished.

\section{Results}

In addition to the above-mentioned benefits, as a result of the tests, "AYA Sport" application has recorded an unprecedented speed of data acquisition and calculations - only 2-3 seconds, while "Strelok Pro" program requires about 15 seconds.

In real-time fire tests, the results shown in Table 1 have been obtained.

Table 1. The accuracy based on distance measurement.

\begin{tabular}{ll}
\hline Distance [m] & Accuracy [\%] \\
\hline 100 & 99 \\
200 & 98 \\
500 & 97.5 \\
1000 & 98.3 \\
\hline
\end{tabular}

\section{Conclusion and Future Works}

5. Conclusion and Future Works

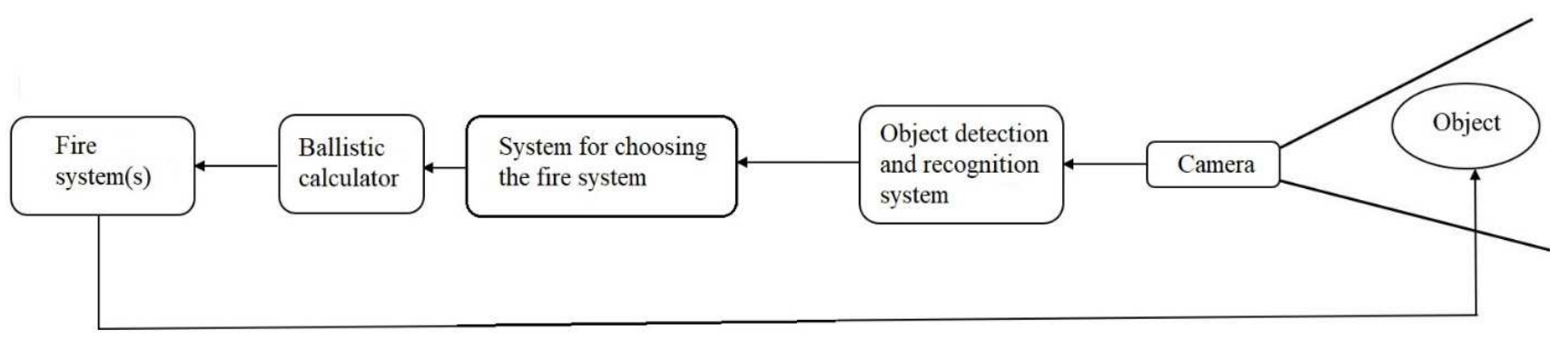

Figure 6. The diagram of distance detection.
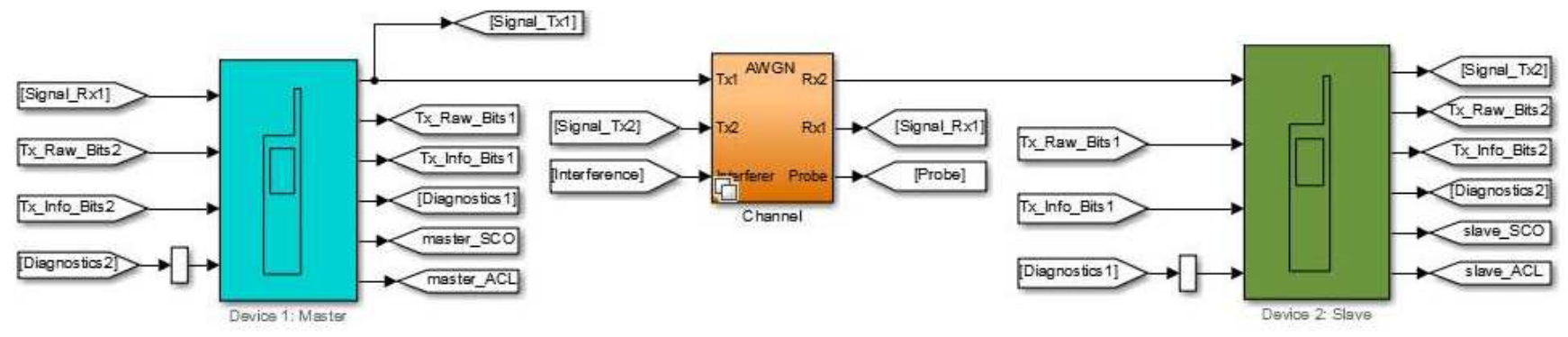

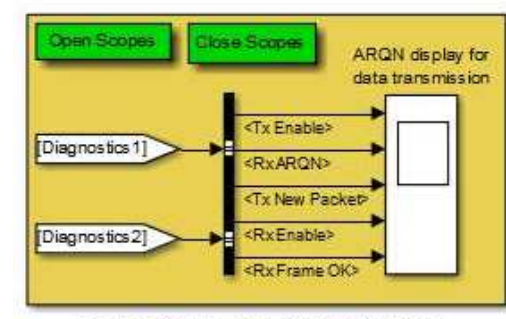

Data Transmission Display

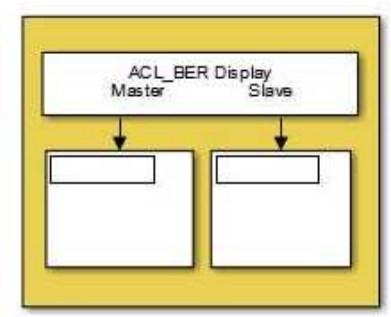

ACL BER Display
Summing up the accomplished work and research, it can be stated with confidence that "AYA Sport" application can become an absolute leader on the ballistic calculation software market in the near future.

Based on the results obtained, it will allow to develop a fully automated general system that will perform objects detection, differentiation and, if necessary, depending on the type of the object, corresponding ballistic calculations [8]. The simplified scheme of the system has the look shown in Figire 6.

At this point, there are different solutions for detecting and deleting the object. For landmine objects, heat and noise response systems have become more widespread. Most use radar for air or water objects. However, the use of the Camera Vision system gives an opportunity to utilize the same system to detect the object in any environment.

Unlike the rest of the system, the above-mentioned system differs from its low cost, easy service and high reliability. However, only detection of objects can not be considered as a complete solution to the problem. That's why the whole system has the following schematic look:

Figure 7. The simplified model of the system for choosing the fire system.

Complete cohesion is a combination of several other subsystems,

1. Camera - a system which is aimed to get the image from the external environment and to transfer it to the image processing subsystem. It should be noted that in order to ensure high efficiency, the camera must provide a high quality image and have wide range visibility.

2. Object detection and recognition system - is aimed to process images received from the camera, searching for objects considered as target samples, and transfer the data to the fire system selecting device. The whole functionality of the webcam is limited to getting the 
image and transferring it to the image processing system. The latter is already endowed with a primary database and trained in data detection and distinction, and decides whether there are any of the searched objects in the received image and if there are any, than which ones. The selected algorithm for the detection of objects, the size of the database used for the preliminary training and the computational power of the system are decisive here, which is directly proportional to the decision-making speed of the image processing system $[9,10]$.

3. The fire system selecting device - Getting information this device first checks the authenticity of it beforehand knowing the image processing system identifier. In the case of a reliable source of information, it pays attention to the exact object that has been detected and whether there is a fire system among those attached to it that can strike that object, considering the type of the weapon and the distance to the object. In the case of such a fire system, all the information obtained from the image processing system, except for the authenticity checking identifier, is reported to the ballistic counter. The simplified model of the system for selecting the fire system is shown in Figure 7.

The developed computer model describes the data transmission procedure between the equipments connected by the Bluetooth network through AWGN channel $[11,12]$. As the transmitted traffic is not sensitive to delays, it has been decided to use the asynchronous communication mode. The Slave Master provides packets to the main device only when the latter has received the corresponding package in the previous time slot. The model uses ARQ mode of data transmission, which allows the distorted packets to not be further processed. The Data Transmission Display subsystem serves for the visualization of that mode.

1. Ballistic calculator - performs the necessary ballistic calculations based on the received information. As ballistic calculations are ready, they are transmitted to the fire system. The ballistic calculator receives information about the object subjected to striking and performs ballistic calculations for a specific fire system. It should be noted that when calculating, air humidity, temperature, wind direction and velocity are taken into account. All of the above data can be input to the ballistic calculator by the operator, as well as automatically, in case of having the necessary external devices. As the calculations are ready, the ballistic calculator transmits them to the appropriate fire system. "AYA Sport" application has been designed according to the requirements for the ballistic calculator, which performs the corresponding calculations within seconds.
2. Fire system- here an automatic rifle or cannon may be used as a unit. The purpose of the system is to strike the target by obtaining the necessary settings.

\section{Acknowledgements}

Thanks to "Hi Tech Gateway" and "ConnectTo Communications" companies for providing all the necessary equipment and permission to share "AYA Sport" architecture and working concept.

Special regards to Armenian military forces for testing the application and sharing the results.

\section{References}

[1] I. Borisov, Russia. [Online] Available at: https://play.google.com/store/apps/details?id=com.borisov.stre lokpro

[2] A. Stepano and A. Lebedinets, "Calculations of external ballistics in firing efficiency studies", in Engineering newsletter, no. 8, 2015, pp. 537-542.

[3] Ja. Shapiro, "External ballistics. rev Moscow (RU)", in State publishing house of defense industry, 1946.

[4] A. Konovalov and Yu. Nikolaev, "External ballistics. rev Moscow (RU)", in Central Research Institute, 1979.

[5] D. Ventsel and Ja. Shapiro, "External ballistics. rev Moscow (RU)", in State publishing house of defense industry, 1939.

[6] A. Aliverdiev, "Calculation of the dependence of the distance of the shot and bullet speed. Forensic examination", no. 3, 2005, p. 49.

[7] A. Karapetyan, Armenia. [Online] Available at: https://play.google.com/store/apps/details?id=com.connectto.a ya_sport

[8] A. Karapetyan, "Testing of the CNN and SSD methods at object detection application. In NPUA annual conference, 2018.

[9] A. Ter-Martirosyan, A. Karapetyan, H. Gomtsyan, "Analyzing the operation principles of camera vision systems", in Proceedings of National Polytechnic University of Armenia, no. 1, 2018, pp. 116-123.

[10] R. Chuikonov, D. Yudin. "Detection of vehicles at the images of country road based on SINGLE SHOT MULTIBOX", in Information Technologies, 2017, no. 4.

[11] "AWGN Channel", in "Math Works", [Online] Available at: https://www.mathworks.com/help/comm/ug/awgnchannel.html.

[12] K. Mhlanga, M. Mafumo, P. Sotenga, etc., "Design and Simulation of a Bluetooth Communication System", in "International Conference on Emerging Trends in Networks and Computer Communications (ETNCC)", 2015. 